\title{
PLANETARY TEMPERATURES IN THE PRESENCE OF AN INERT, NONRADIATIVE ATMOSPHERE
}

\author{
JOHN LESLIE NicOL \\ Department of Physics, James Cook University of North Queensland, Townville, Australia
}

Manuscript received: February 7, 2020

Revised version: August 18, 2020

\begin{abstract}
NicoL J.L., 2020. Planetary temperatures in the presence of an inert, nonradiative atmosphere. Quaestiones Geographicae 39(3), Bogucki Wydawnictwo Naukowe, Poznań, pp. 69-85. 11 figs + attachment.

ABSTRACT: This study considers solid planets at about $300 \mathrm{~K}$ and an inert atmosphere having no interaction with associated radiation. Processes considered include transfer of energy from the surface skin to underlying layers depending on thermal properties. Temperatures of the surface depend on the rates of transfer of energy between soil layers. The atmosphere is warmed at base by contact with the surface, convection and turbulence distributing higher temperatures through the air. Comparisons between theoretical and measured temperatures show a close similarity. Mean planetary temperatures are calculated, depending on thermal parameters and the intensity of light/radiation from the particular solar system.
\end{abstract}

KEY WORDS: planet, inert atmosphere, temperatures, capacity/conductivity, soil, air

Corresponding author: John Leslie Nicol jonicol18@bigpond.com

\section{Introduction}

In our initial considerations, we discuss an uncomplicated planetary surface, consisting of a deep crust of soil overlain by a contacting gaseous atmosphere which has no potential for the emission of electromagnetic radiation or complementary absorption. The solid surface is assumed to be irradiated by a changing sequence of intensities of solar radiation which in general varies semi-sinusoidally on a diurnal basis with equal periods of daylight and night. During daylight hours (which, in the case of Earth, covers a period of $43,200 \mathrm{~s}$ ), the planet's solid surface is warmed from its top, the progression of the temperature increasing downwards into the soil and being described in the analysis by reference to a sequence of thin, clearly defined layers, the warmest being then at the planetary surface and the coolest at the lowest reach of the penetrating warmth. At the same time, the inert atmosphere above, which itself has no visible or infrared absorption/emission bands but is in contact with the soil's surface, is warmed by it through conduction of heat, an effect which is enhanced by the mixing of the warmed air through its lower layers through convection.

While large scale convection is not considered in detail, it could be expected that the wellknown circulation cells would be readily generated through this process, taking account of differing temperatures arising from variations in solar intensities with latitude, in contrast to arguments that are sometimes made (Spencer 2016) that this heating will, in general, simply result in a uniformly heated, stagnant, warm atmosphere.

During the nightly process of radiative cooling from the thin skin forming the surface, the relative temperatures of the lower soil layers change sequentially, the top layer quickly adopting a temperature, $T_{0}$ which is defined by the equilibrium between the rate, $P_{j}^{\prime}$ at which energy can be transferred by conduction between lower layers $j, j+1 \ldots$ of temperatures $T_{j+1}$ and $T_{j}$ respectively, and eventually to the uppermost layer of temperature $T_{0}$ overlain by the surface skin and the rate 
$\mathrm{Pr}$ at which energy is radiated from the surface skin to a cold space, which is given by

$$
\operatorname{Pr}=\sigma T_{0}^{4}
$$

where:

$\sigma=5.67 \times 10^{-8} \mathrm{~W} \mathrm{~m}^{-2} \mathrm{~K}^{-4}$ is Stefan's constant.

Thus we find from the principles of thermal conduction (Look, Alexander 1988):

$$
P_{j}^{\prime}=c_{j}^{\prime}\left(T_{j}-T_{j+1}\right) / \delta
$$

where:

$c_{j}^{\prime}$ is the conductivity of the soil between the centres of layers $j, j+1$, for which examples from a planetary surface indicate a range of values between $0.4 \mathrm{Wm}^{-1} \mathrm{~K}^{-1}$ and $3.0 \mathrm{Wm}^{-1} \mathrm{~K}^{-1}$ (Engineering ToolBox 2003), $\delta$ is the distance between the pair of points of temperatures $T_{j}$ and $T_{j+1}$.

The skin temperature may attain relatively low values for very low conductivities of the soil, the stable cooled layer of air in contact with it providing little heating while maintaining a slowly decreasing temperature through its own lower layers, depending on air conductivity and thermal capacity.

Cooling at night and the differences in temperature between regions of the planet's surface provide differing local pressures causing lateral movement of gas from higher to lower pressures and induce warmer air to rise, providing circulation.

However, here, we are not concerned with major circulation processes which, though very important in studies of the complete atmosphere, do not significantly change the physics of the specific surface effects. Considering the low thermal conductivity of air, it is not difficult to show that while the soil is cooling, there is a considerable difference between the air temperature a very short distance above the surface skin and that of the surface itself except at the point of contact between air and soil, a situation where convection or turbulence are effectively suppressed by the inversion of these temperatures.

During warming of the surface through hours of daylight, energy flows within the system are defined by equilibrium between absorption of radiation of intensity $I_{s} \mathrm{Wm}^{-2}$ from the incoming solar field, the rates of flow of heat $I_{g}$ from the warmed skin to the layer $\mathrm{j}=0$ beneath and to the atmosphere above $I_{a}$, and the surface radiation, $I$, which depends on the temperature of the skin through Eq. (1).

Thus, we may write

$$
I_{s}-I_{g}-I_{a}-I_{r}=0
$$

The magnitude of $I_{s}$, the intensity of sunlight at the surface, obviously depends on the size and brightness of the sun and the sun - planet distance. For Earth, the value of $I_{s}$ is approximately $1368 \mathrm{Wm}^{-2}$, a figure, which according to NASA (2003), varies by about $\pm 1 \mathrm{Wm}^{-2}$ over the 11-year solar cycle, whereas absorption by various components of the atmosphere results in a surface intensity of about $970 \mathrm{Wm}^{-2}$.

Through the rotation of a planet, $I_{s}$ varies semi-sinusoidally, changing between zero and its maximum, in a period representing half of the planet's day. Heat flow to the ground and the air layer above is illustrated in Figure 1; depending

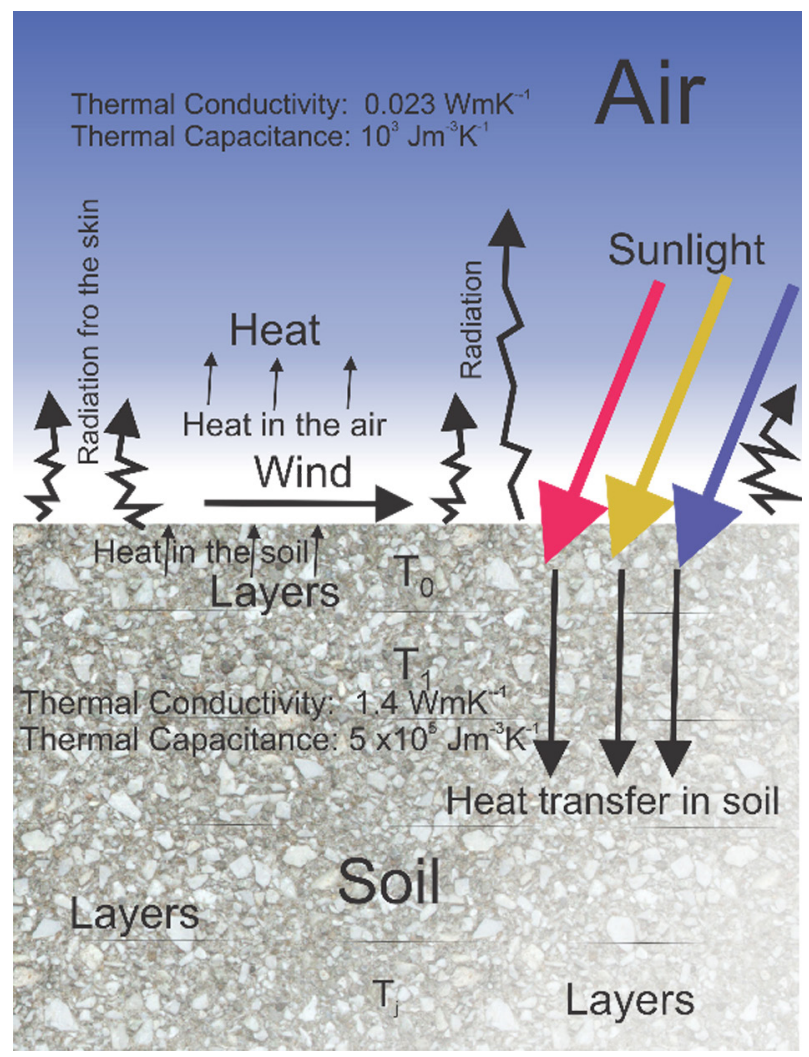

Fig. 1. Energy from the visible sunlight absorbed by the planet's surface raising the temperature of the soil below as the heat is transmitted to lower levels. The air above is also warmed through the contact with the hot surface skin, which also emits energy to space as infrared radiation. 
on the mutual temperature differences, Busby (2015) found an average temperature of $281.8 \mathrm{~K}$ at a depth of $1 \mathrm{~m}$ in the North of Scotland and similarly $285.7 \mathrm{~K}$ in Southern England, temperatures considerably in excess of the surface temperatures.

From the commencement of cooling at night, $I_{s}=0$, thermal equilibrium may be represented by the following equation

$$
I_{g} \pm I_{a}-I_{r}=0
$$

where the flow, $I_{g}$ within the soil is upwards and the direction of $I_{a}^{g}$ is dependent on the relative air or soil temperatures, as indicated by the \pm sign with radiation, and $I_{r}$ again is dependent on $T_{0}$.

Thus, we denote the energy of the lower layers of air and those in the upper segments of soil at any instance of time by

$$
E_{\text {Air }}=\sum_{j=0}^{N} \epsilon_{j}^{a}
$$

and

$$
E_{\text {Soil }}=\sum_{j=0}^{N} \epsilon_{j}^{s}
$$

where:

$$
\epsilon_{J}^{a / s}=\delta_{J}^{a / s} C_{J}^{a / s} T_{J}^{a / s}
$$

- $\delta_{J}^{a / s}$ is the thickness of layer $J$ in either air or soil ( $a$ or $s)$,

- $C_{J}^{a / s}$ is the corresponding thermal capacitance, and

- $T_{J}^{a / s}$ is their temperature.
The flow of heat between these layers depends on the temperature difference, $T_{J}^{a / s}-T_{J \pm 1^{\prime}}^{a / s}$ divided by the distance between the layer centres (often taken as the constant thickness ) multiplied by the thermal conductivity $c_{a J}^{\prime}$ or $c_{s J}^{\prime}$ of the particular material, air or soil, respectively, as defined by Eq. (2). The number of layers and their thickness in each case are chosen to provide an appropriate accuracy as defined by the calculations using a range of numbers to determine the limit where further reduction in the thickness of layers provides no significant change in the results.

Figure 2 presents the temperatures of a warming and cooling soil, the solar radiation falling on the surface periodically, with temperature differences transferring heat between layers either upwards or downwards, following the constraints represented by Eqs (1)-(4). The temperature variation here corresponds to a maximum solar intensity of $1500 \mathrm{Wm}^{-2}$ and is unmodified in this case by the ballast which would be apparent in the presence of an inert atmosphere.

For each pair of adjacent layers of soil denoted by $j$ and $j+1$ and of thickness $\delta$ the power transferred between them, consistent with Eq. (2), is simply

$$
I_{a / s}=c_{a / S J}^{\prime}\left(T_{J}^{a / s}-T_{J \pm 1}^{a / s}\right) / \delta_{J}^{a / s}
$$

These processes are also discussed in some detail by Cheruy et al. (2017).

Obviously, the value of the surface radiation intensity, $I_{s^{\prime}}$ varies very significantly according to the time of day. However, we note that in this clear, inert, atmospheric model, no change is made to any particular time-dependent intensity through the impediment of clouds or aerosols,

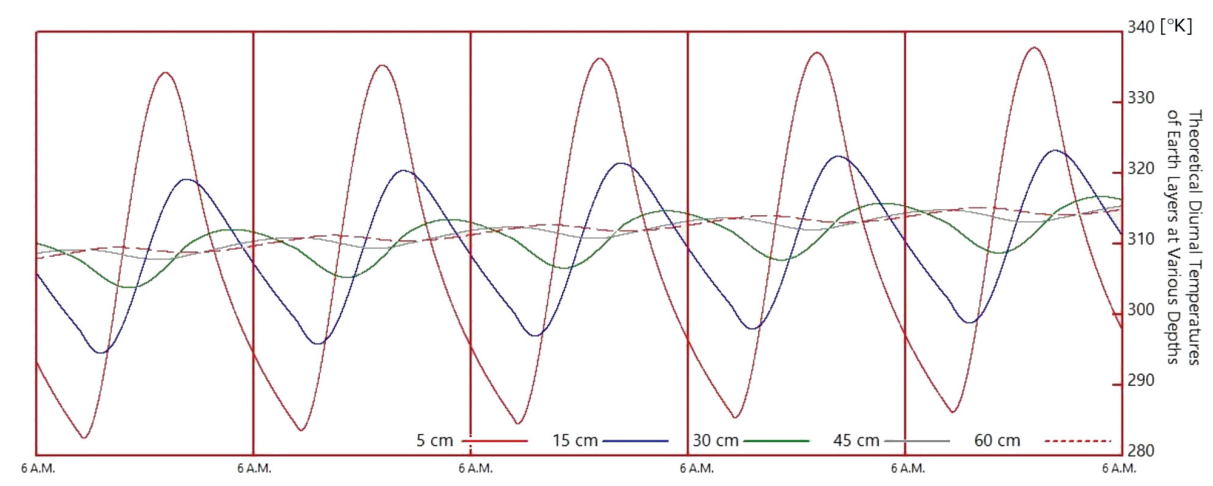

Fig. 2. Diurnal temperature changes at various depths of 5, 15, 30, 45 and $60 \mathrm{~cm}$. Earth conductivity $=1$. Phases of the so-called temperature-waves similar to those shown in the diagram of experimental measurements -

Figure 3. 
while the inert atmosphere has no properties of radiative interactions, neither of absorption nor emission. Thus, in order to understand the outcome of these clearly defined transfers of heat energy, one needs to analyse carefully the details of each of the four processes involved.

It will be shown that the temperatures of the radiating skin depend on equilibria between the independent channels of heat transfer to or from both the underlying conducting layers of the subsoil and the warmer or cooler air in contact with it, the temperature dependent rate of infrared radiation from it defined by Eq. (1) and, in daylight hours, the intensity of the incoming radiation.

\section{Application of Eq. (1)}

The keys to determining the temperature outcomes will depend on the initial influx of power and its subsequent division and transfer as defined by Eqs (1)-(3) with expressions representing the processes defined in these equations. For the hours of daylight at any point on the planet's surface, the radiation intensity may be defined through its angles of latitude and longitude, $\theta_{i}$ and $\varnothing_{j^{\prime}}$ by the expression

$$
I_{s}^{i, j}=I_{0} \cos \left(\theta_{i}\right) \sin \left(\varnothing_{j}\right)
$$

where $I_{0}$ represents the maximum of the solar intensity at the planet's surface.

At each point, the internal heat flows are given by Eq. (8) and at equilibrium, it is required that

$I_{0} \cos \left(\theta_{i}\right) \sin \left(\varnothing_{j}\right)+c_{0}^{i^{s}} \frac{T_{0}^{s}-T_{1}^{s}}{\delta_{I}^{s}}+c_{0}^{\prime a} \frac{T_{0}^{a}-T_{1}^{a}}{\delta_{I}^{a}}+\sigma T_{0}^{s 4}=0$

where: $T_{0}^{a}$ is the temperature of the lowest, very thin, layer of air in contact with the skin of the soil, $T_{0}^{s}$ is that of this surface's skin, $c_{0}^{\text {aa }}$ and $c_{0}^{\text {ss }}$ are the conductivities of the air and soil, respectively, generally expressed in $\mathrm{Wm}^{-1} \mathrm{~K}^{-1}$.

Thus, at any point in time, Eqs (8) and (10) represent the interactions, through the conduction of thermal energy, between an initially arbitrary set of temperatures in a body, the true values of which are determined through the application of these equations to a repetitive sequence of daily warming and nightly cooling until an equilibrium is established. This process, which represents the real effects of continuing cycles of warming and cooling, provides the daily values of temperatures of the layers of air and of the soil, the top layer of soil representing the initial sink for the solar energy and the source from which the radiation component $I_{e}$ emerges.

In this way, we determine the value of $T_{0}$ which satisfies the instantaneous but continuously changing equilibrium which will be established within the very thin surface skin as it receives radiation and effectively distributes its energy depending on the relative temperatures of it and adjacent layers of absorbing material, air and soil, in contact with it, while radiating the remainder to space. An essential criterion that is continuously monitored is the effective emission temperature corresponding to the hypothetical distribution over the planetary surface of the mean value of $T_{0}$ providing a total power of the radiation field which is exactly equal to that of the incoming solar field.

\section{The temperature of the ground}

During each $12 \mathrm{~h}$ of daylight, the temperature $T_{0}$ of the soil's surface at any point varies according to the latitude and longitude of that point, the season of the year and the time of the day. In the artificial, though representative, model of a planet with an inert atmosphere used here, free of cloud and aerosols, the temperatures recur regularly and are determined by the sun's intensity at each time of the day and by the residual energy retained by the air and the soil. Conduction of energy from the solar heated upper layer of the planetary surface to lower depths may be considered in terms of a series of thin layers of soil of equal thickness - in practice each being approximately $1 \mathrm{~cm}$ in depth with conductivities typical of the material being considered, clay or sand, dry or moist. Calculations of energy transfer through the surface material, generally through a depth of $<0.5 \mathrm{~m}$ to match the reality that little or no diurnal change in temperature takes place at depths larger than this figure, involve considerations of the rate of transfer of energy between successive layers. This rate is dependent on the difference between their temperatures, the thermal conductivity of the material and its thermal capacity which defines the temperature changes consistent with the amount of energy transferred between each 
pair of layers. Specifically, the process of transfer of energy through a sequence of layers - for example 30 layers of earth of thickness $\delta_{J}^{s}=0.01 \mathrm{~m}$ to a total depth of $0.3 \mathrm{~m}$ - is carried out through a sequence of calculations from Eq. (8) in the form

$$
\delta T_{s}=c_{s J}^{\prime}\left(T_{J}^{s}-T_{J \pm 1}^{s}\right) / \delta_{J}^{s} C_{c a p}
$$

where :

$\delta T_{\mathrm{s}}$ is the temperature transferred from layer $J$ to layer \pm \pm 1 , each layer being assumed to be of equal thermal capacity $C_{\text {cap }}$ per square metre.

Thus we now have new values:

$$
\begin{gathered}
T_{J}^{s}=T_{J}^{s}-\delta T_{s} \\
T_{J \pm 1}^{s}=T_{J \pm 1}^{s}+\delta T_{s}
\end{gathered}
$$

This process is assumed to take place every second corresponding to the receipt of an associated parcel of energy in the top layer. Repetition of its application over several days leads to the results shown in Figure 2, representing the calculated temperatures at various depths in soil. Experimental values shown in Figure 3 confirm the form of these temperature changes over a period of a day. Similar equations can be written for the layers of air with correspondingly lower values for its heat capacity and thermal conductivity, making allowance for the results from convection and turbulence.

During the hours near midday, and particularly soon afterwards, the temperatures of all components reach maximum values and in the case of the soil - air interface, energy passes from the surface skin to the air above in packages, which are subsequently dispersed by convectional circulation and turbulence as described and discussed by Donner et al. (2016). For the inert atmosphere being considered here, dissipation of energy from the air to the soil can also take place through conduction at the air - soil interface, with transfer being possible only when the temperature of the soil's skin is lower than that of the contacting air. At night, the earth's surface cools continuously through radiation leading simultaneously to a return of energy from the lowest layers of the soil to its surface and to a much lesser extent from the air above.

In the case of radiation from the surface skin as defined by Eq. (1), the value of $T_{0}$, and hence $P_{r}$, is restricted by the limited rate of transfer of energy from lower to higher layers, partially augmented or depleted by heat transfer from or to the air. Thus, in the case of cooling at night, the temperature of the skin will in general be lower than contiguous layers of soil below.

Complementary to the above considerations of the transport of energy through the thermally conducting soil, it is instructive to study the temperature $T_{0}$ of the planet's skin while hypothetically maintaining a given temperature $T_{d}^{s}$ within a much lower layer of soil. In a vacuum, the surface energy is removed by radiation alone and at a rate determined by the Stephan - Boltzmann law, Eq. (1). It is obvious that the power radiated cannot exceed the power that can be delivered to the surface from the lower warmer layer, a process which is described by the sequence of Eqs (11)-(13) and where each temperature, $T_{j}^{s}, \mathrm{j}=0,1,2, \ldots, j_{\text {max }}$ is sustained or corrected by the flow of energy and hence temperature defined by the first of these equations.

In Figure 4, the various surface temperatures in equilibrium, $T_{0}^{s}$, are shown for a range of soil conductivities and lower layer temperatures at the depth of $0.3 \mathrm{~m}$. These calculations reveal perhaps some unexpected results such as that for a very common temperature (see Figs 2 and 3) of $310 \mathrm{~K}$ at that depth, the equilibrium surface

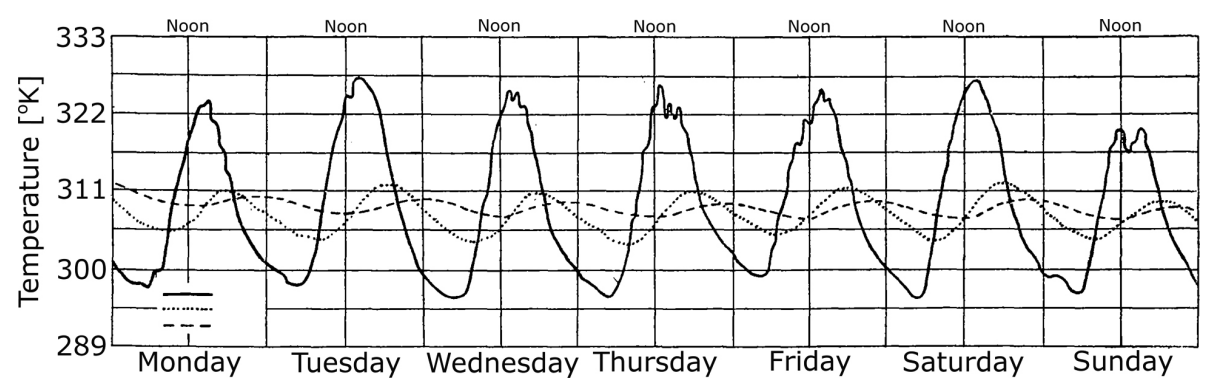

Fig. 3. Continuous measurements of soil temperatures over 7 days by CSIRO, Australia, in January (Summer) 1939 (acc. to West 1952). 


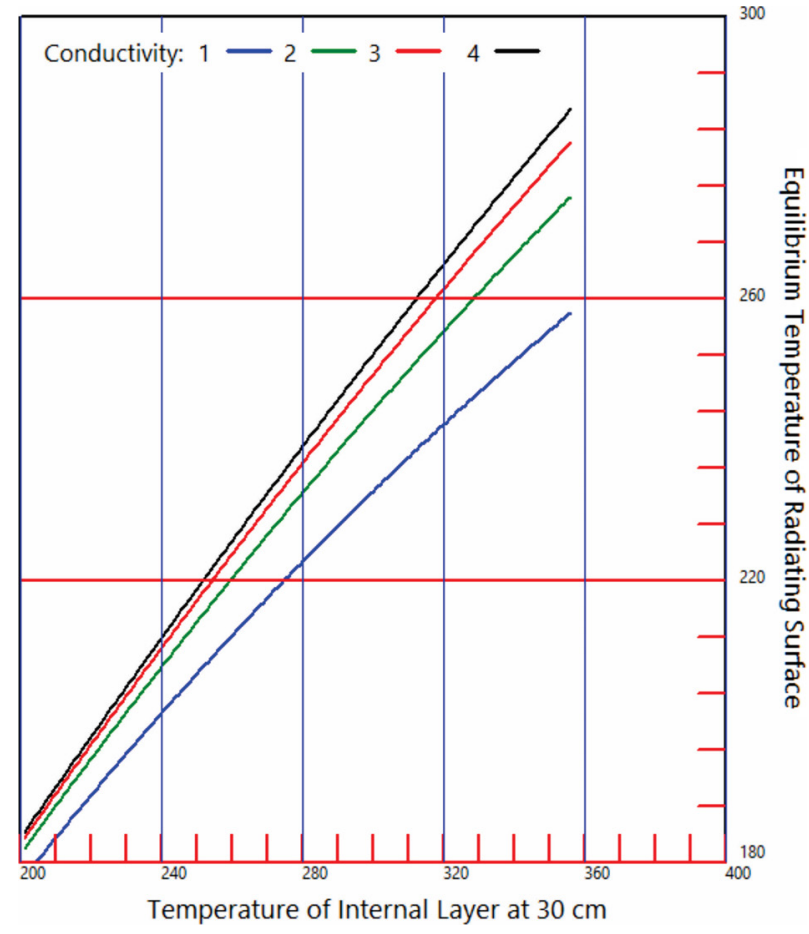

Fig. 4. Temperature of the radiating surface skin of the planet where the temperature of the internal layer at a depth of $30 \mathrm{~cm}$ varies from 200 to $350 \mathrm{~K}$ and for various assumed thermal conductivities of the soil ranging from 1 to $4 \mathrm{Wm}^{-1} \mathrm{~K}^{-1}$.

temperature for a very common soil conductivity of 2.0 is only about $256 \mathrm{~K}$. At higher subsurface temperatures and for higher conductivities, the temperatures are similar to those that are more generally expected near the surface of the earth, bearing in mind that this is an artificial situation constructed to demonstrate the effects of differences arising from lower level temperatures and significant changes in soil conductivities.

Calculations leading to the details shown in Figure 4 show that the required condition of equilibrium between the surface temperature and its gain and loss of energy through the various channels is achieved after the repetition of a sequence of cycles, after which the rate of radiation from the heated surface becomes constant and consistent with the intensity of the sunlight falling on it. In all calculations, this requirement of equality is continually monitored.

\section{The temperature of the air}

The principle of the process of transfer of energy to the air from the warmer surface of the soil's skin is very similar to that which prevails between the pairs of layers defined as making up the soil below and the structure of its temperatures. However, the significantly different characteristics of the air as a fluid and its relatively low conductivity of only $0.0262 \mathrm{Wm}^{-1} \mathrm{~K}^{-1}$ changes significantly the eventual warming and the cycling of its temperature. In the case of warming, the heating of the lower layer of air at the soil skin surface leads to minor convective currents and turbulence significantly enhancing the transfer of heat energy. In cooling, the lowest layer of air is coolest, leading to an inversion which in principle, at least, leads to heat transfer back to the surface of the soil through the low conductivity of the air.

While there appears to be very little available information in the literature regarding temperature differences between the measured air temperature and that at ground level, measurements at Vostock, $78^{\circ}$ South Latitude within the Arctic circle, have been reported by Turner et al. (2009) showing that the air temperature only $2 \mathrm{~m}$ above the ice level is $4-6^{\circ} \mathrm{C}$ above the temperature of the ice below. This is consistent with expectations that the temperature of the surface skin of the earth will in general be lower than the air temperature, particularly under conditions of persistent cooling. However, most work on atmospheric inversions, for example by Smith and Bonnaventure (2017), Kachar et al. (2015), Espín-Sánchez et al. (2018), have more generally considered larger scale events where large temperature differences exist between the ground and heights of hundreds of metres or kilometres. Espín-Sánchez et al. (2018) reported a temperature difference of $9.8^{\circ} \mathrm{C}$ for a change in height of $19 \mathrm{~m}$.

Considering first the warming of air through contact at the interface with the heated planet's surface during daylight hours, the transfer of energy between the two contacting surfaces again depends on their relative temperatures. However, when the soil's surface is hotter, energy flows most rapidly between the two, being enhanced by the fact that, in general, the air is moving across the surface and on becoming heated rises taking the acquired energy with it and being replaced by cooler air to continue the transfer of heat. The maximum possible temperature acquired by the air's lower surface will be equal to that of the soil's skin, which for a hypothetical solar intensity of $1000 \mathrm{Wm}^{-2}$ in the absence 
of absorption by the soil will be $364.42 \mathrm{~K}$ in the thin, lowest layer, a temperature which will be reduced as the parcel of air circulates and mixes its energy with other parts of the atmosphere, while raising the overall temperature of that body of inert gas. In calculations involving air of similar characteristics to that on Earth, the value of the thermal capacity for dry air at constant pressure, $C_{p}$, is $1003.5 \mathrm{~J} \mathrm{Kg}^{-1} \mathrm{~K}^{-1}$ or $1125 \mathrm{~J} \mathrm{~m}^{-3} \mathrm{~K}^{-1}$ (Engineering ToolBox 2003).

In the evening, the cooling of the planet's surface would be expected to lower its temperature below that of the air and its lower layers, forming an inversion (Prigent et al. 2003). In this case, air in contact with it will transfer energy across the interface, tending to warm the soil's skin, and cooling the thin surface layer of air. However, in contrast to the process of heating the air, the cooled, thin, lower layer of gas lies under a warmer body of air, under which conditions no convection will be generated and the cooler air will remain at the bottom, inhibiting, to a large degree, the passage of energy between the two surfaces as the distance from the warmer air to the soil inevitably increases while the temperature difference remains similar. Thus, because of the very low thermal conductivity of the relatively stationary atmosphere $\left(\sim 0.0262 \mathrm{Wm}^{-1} \mathrm{~K}^{-1}\right.$ at 1 bar and $\left.300 \mathrm{~K}\right)$ during the cooler parts of the day and overnight, the transfer of energy from it to the planetary surface is found to be minimal, as discussed earlier. Continuing into the hotter parts of the following day, the air will be subject again to the influx of heat from the ground, a process which must continue until the temperature of the air approaches or is similar to that of the hottest temperature reached by the earth at that point on its surface.

We must emphasise again here that the pure oxygen and nitrogen mixture defined here as the planetary atmosphere cannot radiate or absorb energy from a radiation field which is in equilibrium with temperatures in the range of $250-400 \mathrm{~K}$.

Thus, without the facility for readily losing energy through radiation or by significant contact with even a much cooler surface, the temperature of the whole body of the air must eventually tend towards that of the highest temperature arrived at by the soil surface within the constraints of the requirement for equilibrium within each latitude region of the earth, as outlined earlier. Thus, the air will receive no additional energy during the night while the surface layers of the planet's soil cool at a rate in accordance with that at which heat energy can return to the surface by means of thermal conduction between the arbitrarily defined layers of soil and air. Conductive loss of energy by the air to the cooling soil is significantly minimised by the presence of the stable layer of cooler air juxtaposed between its lowest warmer layer and the soil surface.

Thus, cooling of the entire system is dependent only on radiation from the surface skin, the temperature of which is subject to the limitations imposed by constraints of the conduction of energy from lower layers in the soil and from the atmosphere above as described earlier. While various physical processes are involved in the formation of the well-recognised heat waves, very large hot parcels of air forming in dry desert regions, it is recognised that contact between the air and the hot soil surface provides the most significant contribution (Nairn, Fawcett 2013). By way of demonstration, it is readily observed that the movement of such hot conditions across dry continents of our own planet Earth, where loss of energy from the exceptionally dry air in this particular case, is limited to radiation from greenhouse gases rather than the more generous emissions from water vapour within a humid air mass, and that the high temperatures may persist day and night for several days - or even weeks - in regions over which the air moves, even over much cooler land. Similar comments apply to the cold winds from the polar regions which may be equally dry, and hence substantially unable to absorb the infrared radiation from the warmer earth over which it moves on its way from the poles. Evidence of both of these phenomena, cold fronts and heat waves, is observed almost annually in the North of Europe and Asia, as well as America, Southern Australia and in continents near the tropics which again includes central Australia and Africa. In all of these cases, in the absence of water vapour, the effect of absorption of radiative energy by, or radiation from, greenhouse gases is small and almost ineffective in changing temperatures of the air masses over periods of several days. Hence the incidence of $40^{\circ} \mathrm{C}$ temperatures experienced in Southern Australia $\left(40^{\circ}\right.$ latitude south of the equator) and at Northern Russia's Novosibirsk, $55^{\circ} \mathrm{N}$, only $1000 \mathrm{~km}$ South of the Arctic Circle, with maxima between April 
and September in excess of $31^{\circ} \mathrm{C}$ and winter minima as low as $-40^{\circ} \mathrm{C}$ or $233 \mathrm{~K}$. These air masses are quick to heat but very slow to lose that heat over cooler land while cold fronts from higher latitudes are also slow to change their temperatures when very dry.

Thus, one would expect on these simple physical principles alone that the temperature of the air in the tropics would be equal to temperatures acquired, though perhaps only briefly during the hottest period, by the local soil surface, of say $320 \mathrm{~K}$ and in the mid-latitudes of typical temperatures of $285 \mathrm{~K}$, with much lower temperatures being found characteristically at higher latitudes.

\section{A simple physical analogy}

To further illustrate, the important point being made here in regard to the thermal physics applied to studies of the effects of differing temperatures for the elements on either side of the air - soil interface, we might compare the inert, nonradiative but thermally conducting air with a body of water enclosed in a high-quality Thermos flask from which heat can escape only very slowly. One might heat the water by means of introducing a copper rod of temperature $T$, for a brief time only to some depth into the water, but with much longer periods during which the element is withdrawn. Being insulated by the flask, the water will obviously be heated eventually to a temperature very close to $T$. Were the base of the Thermos flask to be cooled, the water at the bottom will also be cooled but will not rise to warm the main body of the water for a quite significant time, the physical explanation being exactly as that for the atmosphere discussed above.

The effect of the brief injection of heat each midday by the sun will similarly warm the atmosphere towards the highest temperature reached by the planet's surface.

\section{Cooling of a heated solid body}

In considering the warming of the air and the cooling of the planetary surface, the various temperatures of the entire lower surface of the atmosphere and that of the radiating and absorbing skin of the planet itself must be considered. In the latter case, the temperature of the skin depends very little, as we have seen, on the temperature of the air above, once a steady state relationship between these temperatures has been established. At this point, the thermodynamics of the situation is mainly dependent on the incoming radiation, the rate of transfer of energy between the upper layers of the solid material of the planet including the surface skin and the rate of radiation both during daylight and at night from the planetary surface.

By way of a definitive example, we consider a block of soil in the planetary surface which is 1-m deep has a cross-sectional and surface area of one square metre and a uniformly distributed temperature of $300 \mathrm{~K}$. Such a block, being one cubic metre in volume, has a typical thermal capacity of approximately $5 \times 10^{5} \mathrm{~J} \mathrm{~K}^{-1}$, so that its total thermal energy at $300 \mathrm{~K}$ will be $1.5 \times 10^{8} \mathrm{~J}$, and a thermal conductivity of about $1.4 \mathrm{Wm}^{-1} \mathrm{~K}^{-1}$ (Engineering ToolBox 2003). Again, the radiation from its upper surface under ideal nighttime conditions will depend on the temperature maintained on its surface skin by the transfer of heat energy from layers of soil below the surface and on the very low temperature of the clear sky above.

Taking a case with these typical characteristics, we find that by applying a similar algorithm to that used to produce Figure 2, for a period of $12 \mathrm{~h}$ at night, the temperature of the surface is found to fall from the initial value of $300 \mathrm{~K}$ to the much lower value of $232 \mathrm{~K}$ whereas those layers at depths below $50 \mathrm{~cm}$ retain temperatures higher than $282 \mathrm{~K}$, the total energy retained in the sample of soil being about $93 \%$ of its original value. Thus, while the energy is retained to a very large degree in the soil, the temperature of the skin may fall to a low value, significantly below that of the inert atmosphere above, except for the thin stable layer of air at the very bottom, which is in contact with that skin but to which the transport of energy is minimal. This clearly illustrates an important factor in the measurements of temperature of the material of a planet, where, in the absence of other external factors, the skin temperature may at times reach extremely low values even though the soil or other material below this thin surface retains a large quantity 
of heat energy, with similar temperatures but much lower energies, being retained also in the inert atmosphere above.

In particular, assuming again a thermal conductivity of $1.4 \mathrm{Wm}^{-1} \mathrm{~K}^{-1}$, a thermal capacitance of $5.0 \times 10^{5} \mathrm{~J} \mathrm{~m}^{-3} \mathrm{~K}^{-1}$ and with 100 layers of thickness $0.01 \mathrm{~m}(1.0 \mathrm{~cm})$, the sequence of layers from the surface of a cubic metre of soil, numbered 0 , $1,2,3,5,6,7, \ldots, 11, \ldots, 15, \ldots, 50, \ldots, 75, \ldots, 100$ (at 1 $\mathrm{m})$ acquire temperatures after a period of purely radiative cooling of $12 \mathrm{~h}$ of $239.9 \mathrm{~K}, 241 \mathrm{~K}, 243$ K, $244 \mathrm{~K}, 245 \mathrm{~K}, 246 \mathrm{~K}, 247 \mathrm{~K}, 249 \mathrm{~K}, \ldots, 253 \mathrm{~K}, \ldots$, (15) $258 \mathrm{~K},(50) 285 \mathrm{~K},(75) 294 \mathrm{~K}$ and (100)297 K, (the deepest layer at $1.0 \mathrm{~m}$ ) while approximately $93.2 \%$ of the original energy remains in the soil. Clearly the upper layers have cooled rapidly but the energy in the lower layers between 11 and 20 $\mathrm{cm}$ in depth have lost little energy, the constraint being the rate at which this can be transferred across the temperature gradient. This is consistent to a large degree with the known characteristics of dry deserts, which reach very high temperatures during daytime but may become freezing cold at their surface at night. It is noted that the lowest temperature shown from these calculations is $239.9 \mathrm{~K}$ comparable with the lowest temperature recorded in a cold desert of about $233 \mathrm{~K}$ $\left(-40^{\circ} \mathrm{C}\right)$ but for which daytime temperatures may be quite high.

\section{The influence of an inert atmosphere}

As illustrated in Figure 5, and discussed before in detail, the atmosphere above the planetary surface skin forms a layer of inert material in contact with the skin which is capable of absorbing energy by contact and conduction at any

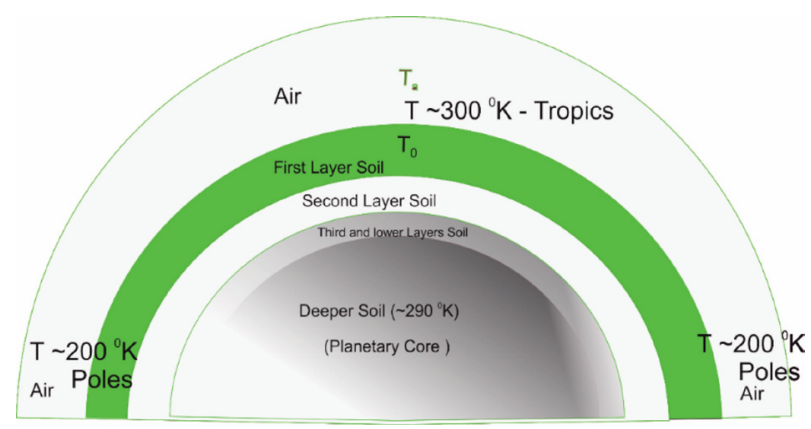

Fig. 5. The planetary soil and inert atmosphere (air) with a higher temperature $T_{\mathrm{a}}$ than solid layers at greater depths. point on the planet's surface in which the temperature is higher than that of the local air. Earlier, the transfer of heat energy across this surface was discussed, being at a much higher rate in the case of the warmer soil than it is for air to return heat to cooler, solid material below.

Though the thermal conductivity of air is relatively low $\left(\sim 0.0263 \mathrm{Wm}^{-1} \mathrm{~K}^{-1}\right)$, contact with hot soil involves only a comparatively thin layer, whereas the heated air above forms minor, but very frequently occurring, points of turbulence, with convection continuously providing a sharp temperature difference between soil and air. This is clearly observed in the formation of mirages even in conditions of high wind when the surface boundary of the air is seen to be continuously heated. Thus for a boundary layer of say roughly $1 \mathrm{~mm}$ and a temperature difference of only $10^{\circ} \mathrm{C}$, the rate of transfer of heat from soil to air would be $0.02 \times 10.0 / 0.001$ (being conductivity $x$ temperature difference / thickness of layer) or $200 \mathrm{Wm}^{-2}$ the temperature difference being assumed to be sustained by the movement of the air, in particular, by upwards convection.

Thus, assuming that the initial temperature of the air is below that of the soil on the side of the planet being warmed by the sun, during a short period of daylight of perhaps only $3 \mathrm{~h}$, during which the temperature difference is assumed to be $10^{\circ} \mathrm{C}$, the air absorbs a decreasing flow of energy with perhaps an average of $100 \mathrm{~W}$ or total energy of $1.08 \times 10^{7} \mathrm{~J}$, which may be taken as a minimum of the likely transfer of energy. Assuming that the total thermal capacity of $1 \mathrm{~m}^{3}$ of air is approximately $1.0 \times 10^{3} \mathrm{~J} \mathrm{~K}^{-1}$, the temperature of a $1000 \mathrm{~m}$ column will rise on average by $10^{\circ} \mathrm{C}$. These calculations are purely illustrative, order of magnitude indicators that over a short period of time, with soil temperatures in the tropical regions of the planet (Earth) reaching maxima as high as $320 \mathrm{~K}$ and the energy corresponding to these temperatures being conveyed by circulation to other cooler regions of the planet's atmosphere, the temperature of the inert atmosphere will reach values which, in general, will be significantly higher than the mean temperature of the planet's surface.

While these theoretical determinations are based on assumptions of values which are only known approximately, the results are very strongly supported by both measurements on Earth as 
discussed below and observations that can be regarded as being experimental in a very large laboratory referred to earlier, where high temperature air remains hot for as long as 7 or 8 days in the form referred to as a heat wave.

\section{Results}

The physical arguments above demonstrate clearly, but without definitive numerical examples, that an inert planetary atmosphere, of sufficient gas density to provide minimal though significant thermal capacity, will invariably result in a continuing temperature of that atmosphere over the period of its day. Because of the more rapid absorption of heat from the surface of the soil with which the air is both in contact and moving laterally because of convection and local turbulence, this temperature would be expected to closely reflect the maximum temperatures repeatedly achieved during daylight hours by points on the soil's surface skin below that atmosphere. Contact between the warmed atmosphere and cooler parts of the skin will result in significantly slower transfer of energy back to the skin from where it may be dissipated deeper into the soil and/or radiated to space. As indicated in earlier discussions of the ground temperatures, this is borne out by experience on Earth, where the temperature of the air rises rapidly in the middle of the day to match that of the soil surface below, but cools less rapidly at night for which its temperature is demonstrably higher than that of the earth's skin as discussed briefly before, the rate of transfer of energy back to the cooler soil being minimal.

This difference develops even though the temperature of the soil's surface is sustained by the upwards transfer of heat energy from lower layers of soil as demonstrated through the results of calculations of skin and air temperatures at various planetary latitudes, the results of which are presented in Figures Aa - Ah of the Appendix. In those diagrams, the blue and red lines represent, respectively, the temperatures on the skin and that of the air at a height of $2 \mathrm{~m}$ above that skin, where the solar intensity in each case varies sinusoidally without impediment and with maximum solar intensities of $200-2000 \mathrm{Wm}^{-2}$. As illustrated at its peak, the incoming solar energy is dissipated to air in very long pulses corresponding to the maximum temperature during each day and similarly to the soil below. It is noticeable that even as the soil has a much higher thermal capacity than the atmosphere, the cooling rate is higher through surface radiation even while the rate is limited, as described above, because of delays in the transfer of heat from deeper layers. On the other hand, as discussed earlier, the rate of transfer of heat downwards from the warmer air to the soil's skin is inhibited by the cooler layer of low conductivity gas between the two components of gas and soil as illustrated broadly in Figure 6 .

A study of the diagrams of the Appendix, Figures $\mathrm{Aa}$ - Ah, shows that they are closely representative of the measurements (e.g. Mass 2011, Marblemount, Washington - Fig. 6) giving the relationship between skin temperatures and 1.2 m-high meteorological measurements of air temperatures. Calculations involving the heating process involving the transfer of heat across the air soil boundary necessarily require some arbitrary assumptions of the temperature gradient but with well-known values of the thermal capacities and conductivities of the soil and the air. Included in this warming process must be an estimate of the rates of energy transfer through small and larger scale convection in which it is clear that even quite slow upwards movements of the air involve the transportation of relatively high powered intensities. For instance, in the case of a $1^{\circ} \mathrm{C}$ rise in the temperature of a cubic metre parcel of air,

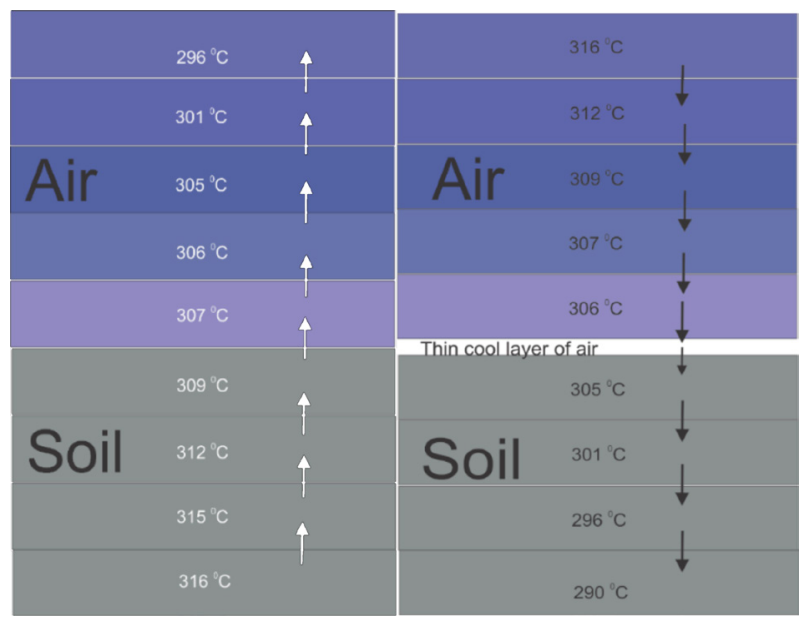

Fig. 6. Model of inert atmosphere above a planetary surface under conditions of slow warming of the solid material from the previously heated atmospheric gases above. Note the thin, stable layer of cooled air shown in white in the right-hand model. 


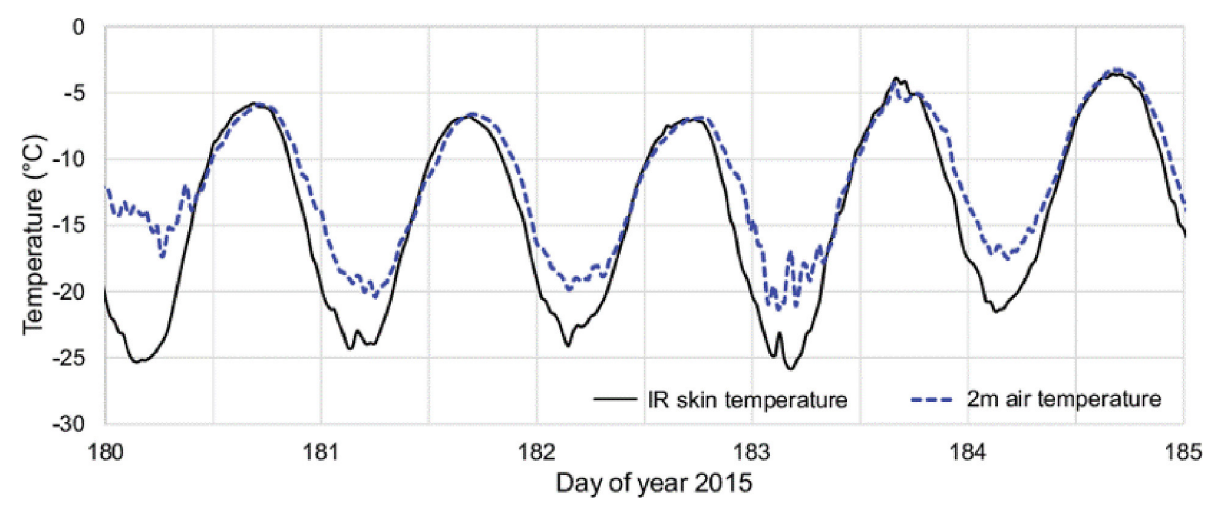

Fig. 7. Time series of temperatures: during a clear sky period near Summit, Greenland. IR skin $2 \mathrm{~m}$ in air (acc. Adolph et al. 2018).

the increase in energy of the air is of the order of $1.0 \times 10^{3} \mathrm{~J}$. Thus air rising hypothetically at this temperature at a speed of, say, only $0.3 \mathrm{~ms}^{-2}$ or $1.1 \mathrm{kmh}^{-1}$ provides an effective energy intensity of $300 \mathrm{Wm}^{-2}$, equivalent to that from total surface radiation, demonstrating that the heating of surface air is usually effective and rapid.

On the other hand and as remarked earlier, cooling of air by a colder skin at the air - soil interface can provide no significant circulation or movement of the air and the change in temperature through this contact is relatively slow, being dependent on the very low conductivity of an increasingly deep layer of nearly stationary air. Thus reasonable estimates of the transfer of heat by these two opposing processes lead to the conclusion that the temperature of the air remains much closer to the maximum temperature of the land as occurs at midday than to the minimum temperature achieved in the early morning by the cooling of the skin, even though the lowest temperature here is restricted by the continuing supply of energy from the warmer, deeper layers below the surface, such as those lying at a maximum depth of about $30 \mathrm{~cm}$. As suggested in Figure 7, the air's temperature at some distance above the ground's surface decays relatively slowly from its maximum while increasing in temperature more rapidly when that of the surface skin has returned to rising.

\section{Discussion}

The physics of heat transfer presented here involves very straightforward and basic thermodynamic principles which are very well established in many textbooks (Look, Alexander 1988). The effect of the deeper levels of the ground in providing a modifying influence on the temperature of its surface skin is remote from the main material sustaining the global temperature, the inert atmosphere, but as shown in Eqs (3) through (8), its characteristics have a significant effect on the temperature of the skin through the effects of its limited thermal conductivity and heat capacity on the allowed rate of internal temperature transfer and hence radiation from the surface of the skin, which is critical to the arguments presented here. Nevertheless, the surface temperature is often comparatively insensitive to the actual magnitude of these soil characteristics. As shown in Figure 4, at temperatures in the vicinity of those found on Earth, $280 \mathrm{~K}$, the doubling of the conductivity leads to a change of only about $4.5 \%$ in that of the skin and much less at higher values of conductivity.

It was also shown that the loss of energy from a $0.3 \mathrm{~m}$ deep soil with an initial temperature of $300 \mathrm{~K}$ throughout and assumed to radiate from its surface to space as a black body is found from very straightforward calculations to cool its surface to a temperature as low as $232 \mathrm{~K}$ in a period of $12 \mathrm{~h}$. At this point, the temperature in the soil at $0.3 \mathrm{~m}$ remains at $297 \mathrm{~K}$, which demonstrates how quickly the surface skin temperature may fall while the total energy in the system remains high, in this case approximately $95 \%$ of the original energy at a uniform $300 \mathrm{~K}$. This is reiterated here as an important concept with regard to the thermodynamics of a solid planet for which the temperature of the surface, and hence the mean skin temperature of the whole surface, depends critically on the heat retained by the upper 
mantle of soil, the thermal capacitance of that soil and by its conductivity.

However, in the context of our discussion, it is the characteristics of the enveloping air that is of most interest, the complementary retention of heat by this material providing a readily measured temperature which is to be compared with that of the maximum acquired by the surface skin of the soil as briefly discussed by Kawashima et al. (2000), Prigent et al. (2003), Mass (2011), Osibanjo (2016) and Adolph et al. (2018).

The results of calculations of skin temperatures and those of the air above shown in Figures Aa-Ah of the Appendix illustrate that the highest temperature achieved by the air in regions close to the ground is equivalent to the highest temperature reached by the soil surface over the daylight hour period - $24 \mathrm{~h}$ on Earth - consistent with measurements shown in Figure 7 at Summit, Greenland, during a clear sky period (Adolph et al. 2018) and Figure 8 at Marblemount (Mass 2011). However, it is obvious that direct experimental comparisons with these results are not generally possible.

The actual intensity of the sunlight at Summit $\left(72^{\circ} \mathrm{N}\right)$ is not known but using the cosine of the angle of the sun at that time of year, about $48.5^{\circ}$ $\left(72-23.5^{\circ} \mathrm{N}\right)$, provides intensities leading to temperatures near midday and during the $24 \mathrm{~h}$ of measurements similar to those shown in the diagram with a maximum of about $-0.5^{\circ} \mathrm{C}$. From this, the form of the relationship between the temperatures of interest, that of the surface skin, in this case ice, and the temperature of the air at a height

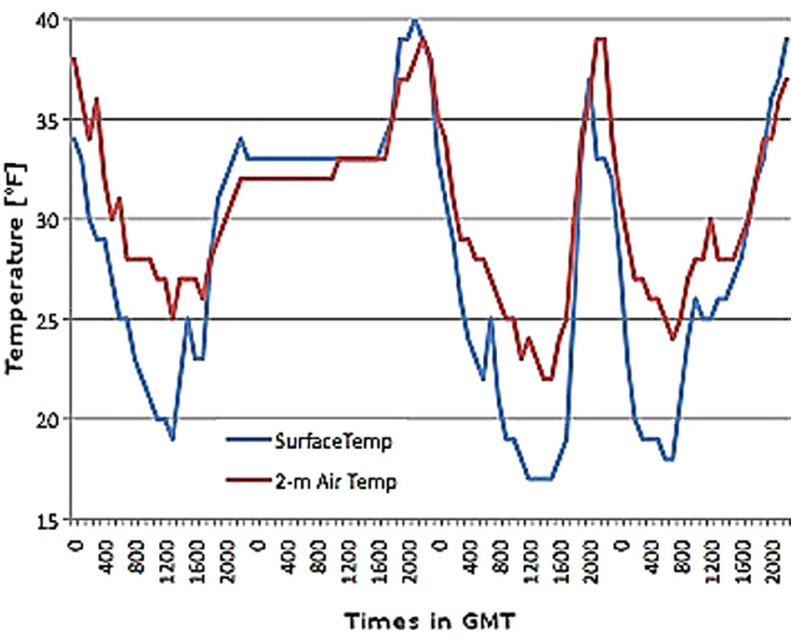

Fig. 8. A comparison of the surface and air temperatures at Marblemount, Washington. of $2 \mathrm{~m}$ above it, is very similar indeed to those shown in Figure 8 at Marblemount, Washington (Mass 2011), and in our own calculations. From these measurements it is useful to emphasise that at the point of maximum skin temperature, the air temperature at a height of about $2 \mathrm{~m}$ above is found to acquire a similar, high temperature and in particular is well represented by the temperatures shown in Figure Af of the Appendix. In both diagrams, the temperature of the air begins to rise earlier in the day relative to the time of the peak temperatures, becoming equal to that of the skin's characteristics - the temperature of the ice at Summit.

Thus, the measurements very usefully confirm that the transfer of heat from the hot skin of the planet when the sun is overhead provides the most significant contribution to the temperature of the air, as discussed earlier, through a combination of heating by contact at the interface and convection as the heated air rises in mass or by way of smaller systems of turbulence. In a hypothetical context, commencing with a body of air of temperature zero, a sequence of bursts of significant heating at this point followed by periods of redistribution through the air mass, but with little allowable return of energy to the planetary surface, the temperature of the air rises asymptotically until it is in equilibrium with the higher surface temperatures, being little influenced by the cooler surface as also described above i.e. the air temperature rises consistently to be equal to the highest temperature of the solid surface below it. When that surface cools, the air retains a significantly higher temperature. Unfortunately, no similar measurements appear to be available for tropical regions.

In Figure 9 (Kawashima et al. 2000), we show a similar relationship between the air and soil skin temperatures but with a different representation. In each case, the low temperature region towards the origin of the plots represents the cooling and cooled surfaces at a significantly lower temperature compared to that in Figure 8. At higher temperatures - around $0^{\circ} \mathrm{C}$ - the air and soil surfaces share a similar value of this temperature.

In Figure 10, the final results of this analysis giving the mean temperatures across the planetary surface for the skin of the solid surface and that for the air above it resulting from significant absorption of energy at periods of maximum 

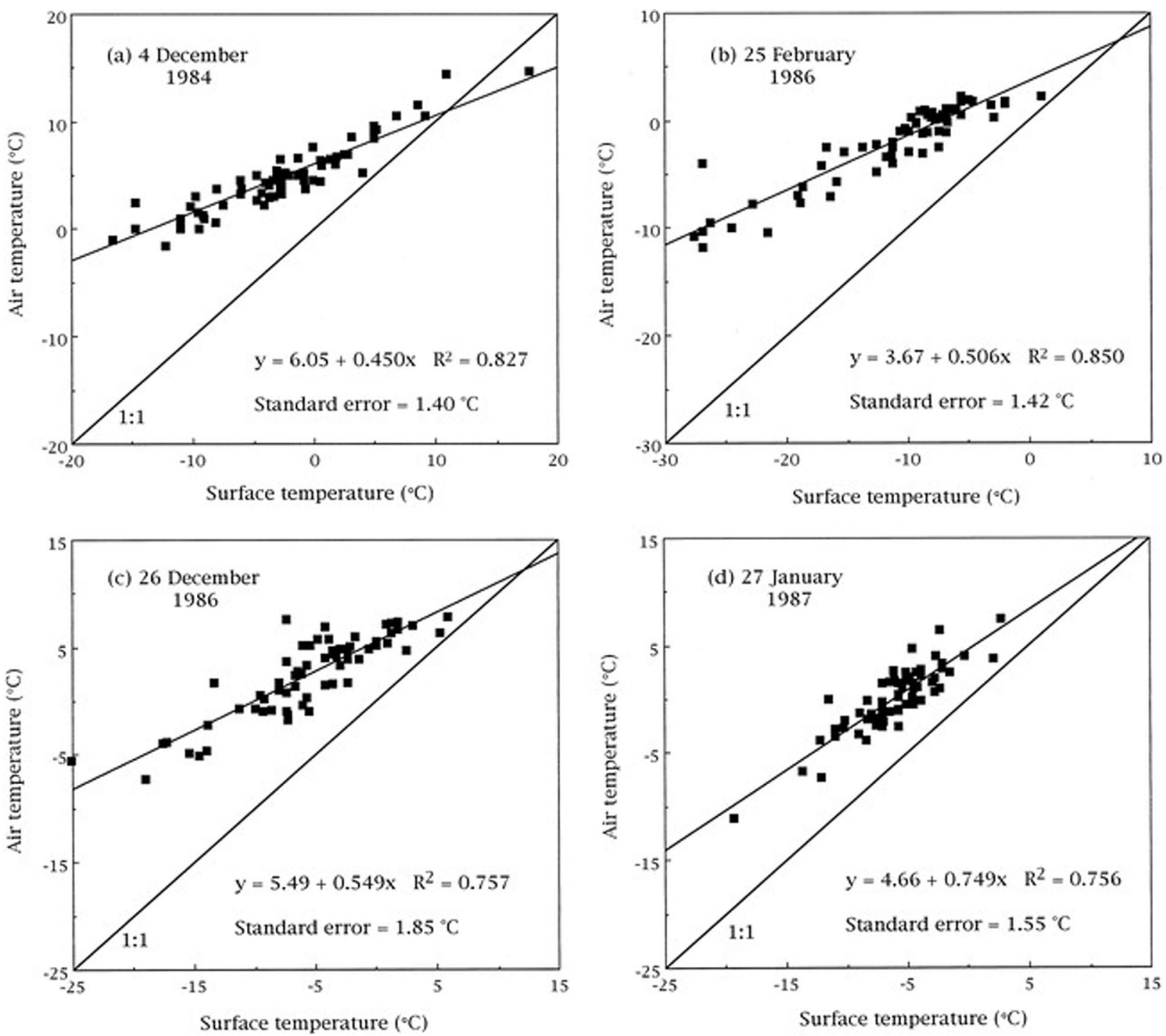

Fig. 9. Relations between surface temperature and air temperature on a local scale during winter nights (acc. to Kawashima et al. 2000).

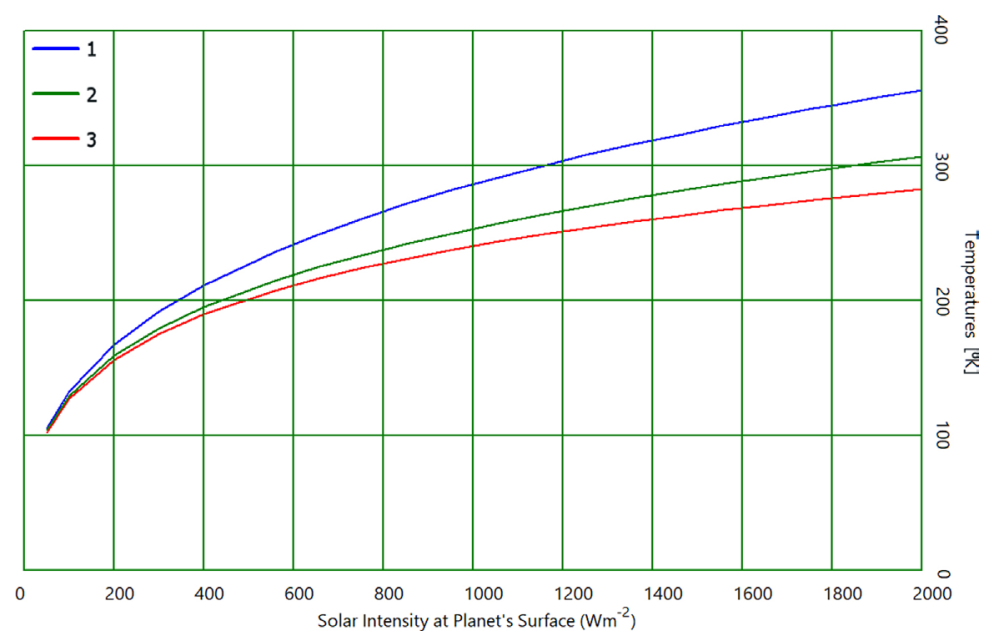

Fig. 10. Characteristic temperatures of a solid planet with an inert atmosphere.

1 - mean air temperature approximately $1.2 \mathrm{~m}$ above the surface skin, 2 - effective emission temperature of the surface skin and 3 - mean temperature of the surface skin. 
surface temperature - midday at each location are shown. The blue line shows the atmospheric temperature which is characteristic of a height of about a metre or more above the skin surface of the solar heated soil, the air's temperature depending largely on the value of the skin temperature's maximum, with lesser dependence on its average over the period of a planetary day. Its continued increase relative to the skin temperatures reflects that of the maximum surface temperature which follows increases in the solar intensity, albeit non-linearly. The green line showing the effective emission temperature of the skin, depends totally on the energy received from the sun and its value for our model of the soil and atmosphere, is required to be exactly equal to that temperature which, when distributed uniformly over the planetary surface, will radiate the same total power as is received from the sun. On the other hand, the average skin temperature (red) shows the result of taking the mean of the surface temperature which varies as the distribution of the energy varies over the surface and is invariably less than, or at most equal to in the hypothetical case of a totally uniform temperature distribution, the effective emission temperature.

\section{Conclusion}

From the results of this analysis that takes account of the retention of heat by the soil of the planet and where present, an inert atmosphere, we see that the temperature which may be defined by that of the air at heights between 1.2 and $2 \mathrm{~m}$ above the skin of the soil's surface is controlled to a very large extent by the maximum temperature acquired by that surface skin through the conduction of heat across the air soil interface. The essential feature of this work has been to demonstrate that the mean temperature of the planet, represented by the lowest parts of its atmosphere, at each latitude is closely related to that of the highest temperatures acquired at midday by the surface skin of the hemisphere during the period when it is facing the sun. Thus, it is very dependent on the maximum value reached and being inert, by our definition here, does not cool by means of radiation. For this reason, the temperature of the whole of the inert atmosphere depends largely on the maximum temperature pulses from the soil surface, received by it during hours of daylight.

The temperatures of the skin and the air also vary significantly with latitude; from all of which one may determine their mean skin temperatures as well as the effective emission temperature of the planet's surface as defined by the IPCC in its considerations of large-scale distributions of atmospheric temperature.

From this we find that the mean temperature of the air at low levels of a few metres - generally 1.2-2 $\mathrm{m}$ above the surface of the soil - is more stable than that of the surface skin and in general significantly higher than the surface temperature. Reports of inversions such as Turner et al. (2009) and Osibanjo (2016) demonstrate that these higher temperatures also extend to significant heights as is also demonstrated by the satellite measurements of radiation from the upper atmosphere and shown in Figure 11 from measurements over Vostock (Turner et al. 2009), for a height above the soil surface skin of approximately $5.5 \mathrm{~km}$. The inert atmosphere discussed here is not subject to many of the effects arising from greenhouse gases and in particular water vapour which can be observed in the atmosphere of earth leading to a complete lack of radiation of energy and to significant changes in the potential for circulation, providing for a warm, stable cover over the soil.

In considering the role played by the deeper soil which also acts as a ballast retaining energy, sequentially absorbing and releasing energy from and to the active upper layer while virtually controlling the temperature of its surface through the limiting of the rate at which energy can flow though the soil, we find that the variation in temperature of deeper layers can be represented in

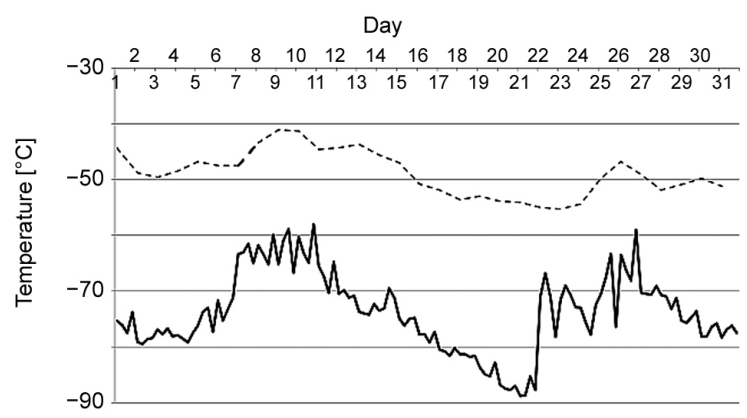

Fig. 11. The Vostok surface (solid line) and $500 \mathrm{hPa}$ (dashed line $\sim 5500 \mathrm{~m}$ ) temperature records for July 1983 (acc. to Turner et al. 2009). 
the form of a wave in time as shown in Figure 2 consistent with experimental measurements, the results of which are demonstrated in Figure 3.

Finally, of most significance are the results shown in Figure 10, representing the three components of the planetary temperatures determined by different values of the solar intensity.

\section{Future work}

The results presented here, while involving much careful analysis, consider only the behaviour of temperatures within and in close proximity to the surface of the soil. Major movements of the atmosphere involving circulation of air between the tropics and the cooler regions of the surface have not been considered, which provide for a rich tapestry for future interesting and complex analysis. However, as a general observation, the convection of warmer air from the tropical regions via circulation cells such as the Hadley Cell of Earth's atmosphere would be expected to transfer warmer air closer to the poles, where it will somewhat slowly transfer heat to the cooler surface of the soil. However, as illustrated in Figure 11, the air above that surface remains warmer and continues to contribute to the total heat content of the atmosphere. In the case of a totally inert system without the radiative cooling from water vapour or greenhouse gases, the mean temperature would be expected to remain even higher through the transport of energy to higher latitudes on any planet. These calculations and others concerning aspects of the current analysis would usefully improve the analysis and the results.

Further critical measurements of the relative temperatures of the soil skin and the air above, such as those shown in Figures 7-9 and 11, should also be carried out in hot, dry tropical regions of Earth where the absence of water vapour renders the atmosphere to be very near to being an inert gas mixture.

\section{Acknowledgements}

I wish to acknowledge with thanks the very significant assistance I have received from the Editors of this journal in preparing the above article in a form suitable for publication.

\section{References}

Adolph A.C., Albert M.R., Hall D.K., 2018. Near-surface temperature inversion during summer at Summit, Greenland, and its relation to MODIS-derived surface temperatures. The Cryosphere 12(3): 907-920. DOI 10.5194/ tc-12-907-2018.

Busby J., 2015. UK Shallow ground temperatures for ground coupled heat exchangers. Quarterly Journal of Engineering Geology and Hydrogeology 48(3-4): 248-260. DOI 10.1144/ qjegh2015-077.

Cheruy F., Dufresne J.L., Aït Mesbah S., Grandpeix J.Y., Wang F., 2017. Role of soil thermal inertia in surface temperature and soil moisture-temperature feedback. Journal of Advances in Modeling Earth Systems 9(8): 2906-2919. DOI 10.1002/2017MS001036.

Donner L.J., O’Brien T.A., Rieger D., Vogel B., Cooke W.F., 2016. Are atmospheric updrafts a key to unlocking climate forcing and sensitivity? Atmospheric Chemistry and Physics 16(20): 12983-12992. DOI 10.5194/acp-16-129832016.

Engineering ToolBox, 2003. Thermal conductivity of selected materials and gases. Online: www.engineeringtoolbox. com/thermal-conductivity-d_429.html (accessed 4 January 2020).

Espín-Sánchez D., Conesa-García C., Castejón-Porcel G., 2018. Temperature inversions due to warm air advections at low levels: significant thermal contrasts in the Vega Media of the Segura River (Southeast Spain). In: Daniels J.A. (ed.), Advances in environmental research. Nova Science Publishers, Inc., Universidad de Murcia, Murcia: 139-177.

Kachar H., Mobasheri M.R., Abkar A.A., Zadegan M.R., 2015. Detection and modeling of temperature inversion in the atmosphere using MODIS images (Case Study: Kermanshah). The International Archives of the Photogrammetry, Remote Sensing and Spatial Information Sciences XL-1/W5: 357-363. DOI 10.5194/isprsarchives-XL-1-W5-357-2015.

Kawashima S., Ishida T., Minomura M., Miwa T., 2000. Relations between surface temperature and air temperature on a local scale during winter nights. Journal of Applied Meteorology 39: 1570-1579. DOI 75/1520-0450(2000)039<1570:RBSTAA>2.0.CO;2.

Look D.C., Alexander G., 1988. Engineering thermodynamics. SI Edition. Springer, Van Nostrand Reinhold (International) Co. Ltd. DOI 10.1007/978-94-010-9316-3.

Mass C., 2011. Surface, air, and soil temperatures: the differences can be large and important. Online: cliffmass.blogspot. com/2011/12/surface-air-and-soil-temperatures.html (accessed 4 January 2020).

Nairn J., Fawcett R., 2013. Defining heatwaves: heatwave defined as a heatimpact event servicing all community and business sectors in Australia. CSIRO and the Bureau of Meteorology CAWCR Technical Report No. 060.

NASA [National Aeronautics and Space Administration], 2003. The inconsistent Sun. Online: science.nasa.gov/ science-news/science-at-nasa/2003/17jan_solcon (accessed 24 January 2020).

Osibanjo O., 2016. Investigation of the influence of temperature inversions and turbulence on land-atmosphere interactions for rolling terrain. MS Thesis, University of Nevada, Reno. Online: hdl.handle.net/11714/2257 (accessed 25 January 2020).

Prigent C., Aires F., Rossow W.B., 2003. Land surface skin temperatures from a combined analysis of microwave 
and infrared satellite observations for an all-weather evaluation of the differences between air and skin temperatures. Journal of Geophysical Research 108: ACL 5-1-513. DOI 10.1029/2002JD002301.

Smith S., Bonnaventure P., 2017. Quantifying surface temperature inversions and their impact on the ground thermal regime at a high arctic site. Arctic Antarctic and Alpine Research 49(1): 173-185.

Spencer R., 2016. The warm earth: greenhouse effect, or atmospheric pressure? Online: www.drroyspencer.com/2016/07/ the-warm-earth-greenhouse-effect-or-atmospheric-pressure/ (accessed 4 January 2020)

Turner J., Anderson P., Lachlan-Cope T., Colwell S., Phillips T., Kirchgaessner A., Marshall G.J., King J.C., Bracegirdle T., Vaughan D.G., Lagun V., Orr A., 2009. Record low surface air temperature at Vostok station, Antarctica. Journal of Geophysical Research 114 D24102: 1-14. DOI 10.1029/2009JD012104.

West E.S., 1952. A study of the annual soil temperature wave. Australian Journal of Scientific Research Ser. A: Physical Sciences 5: 303-314. 


\section{Appendix}
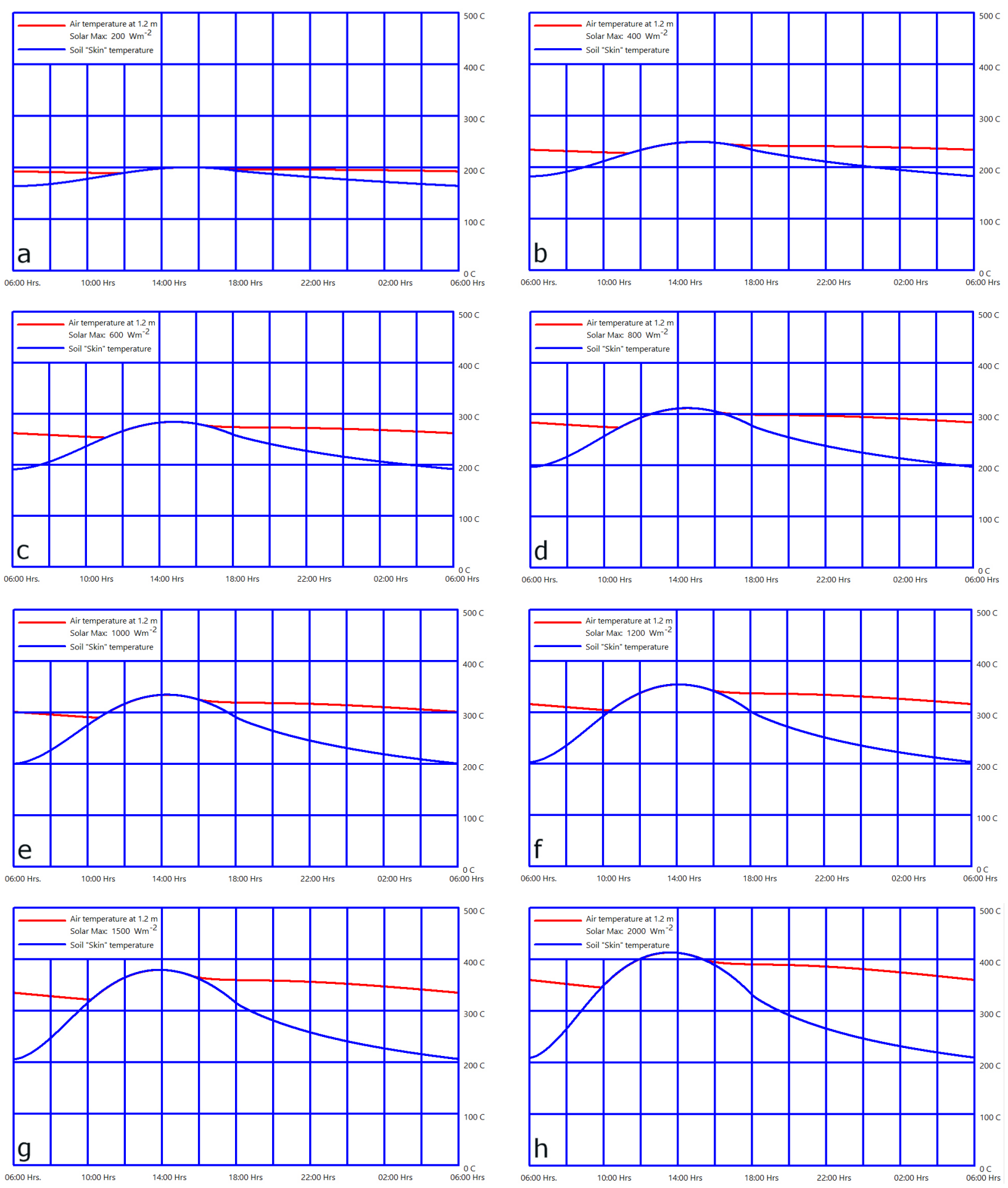

Figure A. The temperature of the surface skin of the earth (blue) and of the air at $1.2 \mathrm{~m}$ above the ground (red) over $24 \mathrm{~h}$. The air is assumed to have a thermal conductivity of $0.03 \mathrm{~W} \mathrm{~m}^{-1} \mathrm{~K}^{-1}$ and a thermal capacitance of $1.006 \mathrm{~kJ} \mathrm{Kg}^{-1} \mathrm{~K}^{-1}$.

For a maximum solar intensity of: a $-200 \mathrm{Wm}^{-2}, \mathrm{~b}-400 \mathrm{Wm}^{-2}, \mathrm{c}-600 \mathrm{Wm}^{-2}, \mathrm{~d}-800 \mathrm{Wm}^{-2}, \mathrm{e}-1000 \mathrm{Wm}^{-2}$, $\mathrm{f}-1200 \mathrm{Wm}^{-2}, \mathrm{~g}-1500 \mathrm{Wm}^{-2}$ and $\mathrm{h}-2000 \mathrm{Wm}^{-2}$. 